\title{
The role of nanomedicine in the imaging and therapy of thrombosis
}

"...targeted nanomaterials may serve as excellent platforms on which to develop multifunctional agents for the unified detection and treatment of this disease."

\section{KEYWORDS: imaging $\approx$ iron oxide " therapy $\approx$ thrombolysis $\approx$ thrombosis}

Hemostasis is the mechanism by which the body prevents blood loss. In its absence, even the smallest trauma would result in the need for immediate intervention to prevent life-threatening hemorrhage, as is seen in coagulation disorders, such as hemophilia. Thus, the formation of blood clots (thrombi) within vessels is a beneficial process as it prevents blood loss. However, in contradistinction to its beneficial effects to limit uncontrolled bleeding, exaggerated clotting can lead to pathological thrombus formation, which, depending on its location, may result in significant morbidity and mortality. Over a century ago, Virchow described three underlying factors in the pathogenesis of thrombosis: stasis, hypercoaguability and vascular injury. On the arterial side, thrombosis (often superimposed on atherosclerotic lesions and usually platelet rich) leads to end-organ ischemia, causing syndromes such as stroke, myocardial infarction and sudden cardiac death. On the venous side, red blood cell- and fibrin-rich thrombi cause the highly morbid deep venous thrombosis syndromes, as well as the potentially fatal syndrome of pulmonary embolism. Regardless of arterial or venous origin, the rapid diagnosis and treatment of pathological thrombosis is of the utmost importance, as restoration of vascular patency is associated with the best prognosis. In this regard, targeted nanomaterials may serve as excellent platforms on which to develop multifunctional agents for the unified detection and treatment of this disease.

\section{Nanoparticles \& the detection of thrombosis}

Thrombus formation is initiated by damage to the endothelial lining of the blood vessels, thereby exposing the subendothelium to coagulation proteins and platelets, which adhere and form the initial hemostatic plug. This clot is subsequently strengthened through the deposition of insoluble fibrin and crosslinking by coagulation factors. Accordingly, there exists a strategic number of targets for which thrombus imaging agents can be developed.

"The diagnostic capabilities of fibrintargeted nanoagents may therefore allow for a better tailoring of therapeutic strategies."

Although thrombosis imaging using targeted nanoagents is indeed possible, the first question that should be asked with regard to their development is as follows: are these techniques going to supplant current clinical diagnostic approaches? When a patient arrives in the emergency room or clinic and a thrombosis syndrome is suspected, a battery of tests are performed. For example, compression ultrasound can be utilized to identify the presence of deep vein thrombosis, while the diagnosis of stroke is confirmed by a combination of $\mathrm{x}$-ray computed tomographic and MR imaging of both arterial vessels and the downstream injured brain tissue. These imaging modalities are rapid, routine and clinically useful, giving functional information about clot location, the size of the potential tissue injury and local blood flow. It may thus be difficult for targeted nanoagents to improve upon the current gold standard of clinical diagnostics often possessing sensitivities and specificities of $85-90 \%$ plus. Yet, these agents may be useful in an adjuvant manner, as they provide molecular and/or cellular information about clot composition and clot age and allow for the determination of the optimum therapeutic strategy.

Numerous small molecule-based imaging strategies have been developed for molecular imaging of thrombogenesis, including

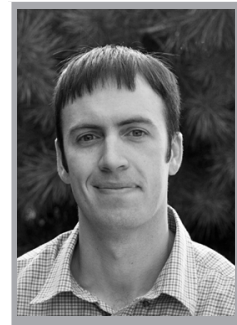

Jason R McCarthy

Author for correspondence: Center for Systems Biology,

Harvard Medical School \& Massachusetts General Hospital, 185 Cambridge Street, Suite 5.210, Boston, MA 02114, USA

Tel.: +16177269218

Fax: +16177265708 jason_mccarthy@hms.harvard.edu

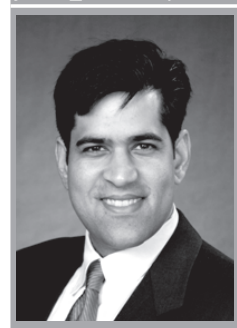

Farouc A Jaffer

Cardiovascular Research Center, Cardiology Division, Harvard Medical School \& Massachusetts General Hospital, Boston, MA, USA 
peptides and antibodies labeled with fluorophores or radionuclides. Yet, few thrombusfocused nanoparticle-based approaches have been reported. Wickline and Lanza of the Cardiovascular Division at the Washington University School of Medicine (MO, USA) have reported extensively on the targeting of perfluorocarbon nanoparticles to clots via antifibrin antibodies. These particles were highly capable of detecting thrombi via ultrasound imaging [1] or by MRI when gadoliniumlabeled [2,3]. A fibrin-specific peptide-targeted nanoagent based upon magnetofluorescent crosslinked dextran-coated iron oxide (CLIO) nanoparticles has also been reported and shown applicability in the optical detection of clots in vivo [4]. Although these materials would prove useful in the diagnosis of thrombosis, their real utility may be in determining the amount of fibrin, the main target of clinical fibrinolytic reperfusion agents, present in a clot. For instance, venous clots are normally more fibrin rich, while arterial thrombi contain more platelets. Thus, the treatment of venous thrombi relies more upon the administration of thrombolytic drugs, while those in arteries respond best to a combination of antiplatelet and thrombolytic therapies. The diagnostic capabilities of fibrin-targeted nanoagents may therefore allow for a better tailoring of therapeutic strategies.

\section{"Nanomedicine ... is well-positioned to play an increasingly larger role in cardiovascular disorders."}

The presence of certain coagulation factors, particularly activated factor XIII (FXIIIa), seen as a hallmark of acute thrombi and represents another useful application for thrombosistargeted nanoengineering. FXIIIa crosslinks fibrin, thereby stabilizing the clot, as well as covalently conjugating $\alpha_{2}$-antiplasmin into the thrombus, augmenting resistance to fibrinolytic agents. Thus, the imaging-based detection of FXIIIa activity could be used to determine the biological age of the thrombus and its lysability, as its activity decreases over time. The feasibility of imaging FXIIIa has been demonstrated using a peptide-targeting sequence derived from the $\mathrm{N}$-terminus of $\alpha_{2}$-antiplasmin and its conjugation to both a fluorophore and a nanoparticle $[4,5]$. In vivo, these agents readily accumulate within acute lesions, having been crosslinked into the fibrin meshwork by the active coagulation factor and provide high target-to-background ratios. Experiments in older clots have shown a time-dependent decrease in enzymatic activity.

Another area of interest is the role of inflammation in vessel recanalization after thrombosis. This is particularly important in venous thrombosis, as it is often viewed as an inflammatory process, with leukocytes and neovascularization playing significant roles in its resolution. This process has been investigated in murine models of resolving thrombus in a multiplexed manner using three spectrally distinct optical imaging agents, including fluorescein-labeled dextran, which can be used to determine thombus permeability, magnetofluorescent CLIO, which is readily internalized by phagocytes and an activatable matrix metalloproteinase (MMP) sensor, specific for MMP-9 [6]. As opposed to previous ex vivo histological studies, this triad of agents was able to simultaneously examine a number of biological factors relevant to thrombus resolution and demonstrate that thrombus permeability correlated well with both MMP and macrophage activity. Although this study was only a first step, the ability to clinically assess inflammation and thrombus permeability may give insight into the course of thrombus resolution and direct the appropriate therapeutic strategy to result in optimal patient outcomes.

\section{Nanoagents for the treatment of thrombosis}

When pathological thrombosis is present, rapid vessel recanalization is imperative to prevent further tissue damage or death, or long-term disability. Reperfusion is accomplished via the administration of fibrinolytic agents, such as exogenous plasminogen activators (PA), which convert the proenzyme plasminogen into an active enzyme, plasmin, which is capable of cleaving fibrin into soluble degradation products. Unfortunately, thrombolysis using PAs is not always effective. In fact, $20 \%$ of infarct-related arteries fail to open, while an additional 15\% of reperfused arteries reclose [7]. Furthermore, there are severe bleeding consequences associated with the use of thrombolytics, including intracerebral hemorrhage, which has been found to occur in $1 \%$ of patients receiving a PA for myocardial infarction [8] and $6 \%$ of patients receiving a PA for stroke [9]. The delivery of these therapeutic moieties to sites of action via targeted nanoagents may thus mitigate these deleterious side effects via a reduction in the dose required to affect efficient lysis (or reduction of off-target effects) and the fact that 
most nanomaterials do not readily translocate through the blood-brain barrier. This last point is exemplified by the amount of research that has been undertaken to address nanoagents to the brain for a variety of pathologies, including cancers and Alzheimer's disease.

In their simplest form, iron oxide-cored nanoagents bearing PAs (urokinase and recombinant tissue plasminogen activator, rtPA) have been targeted to thrombi magnetically and demonstrated increased thrombolytic potential versus their free PA counterpart $[10,11]$. Active targeting strategies have also been employed, including the fibrin-targeted perfluorocarbon nanoparticles described above [12]. These streptokinase-modified particles exhibited a fibrinolytic efficacy 1000 -fold greater than the free drug in vitro. The authors took this system one step further, using a urokinase-modified analog for the targeted treatment of stroke [13]. Thrombolytic nanoagents targeted to acute, and thus more lysable, thrombi, based upon the conjugation of rTPA to FXIIIa-targeted CLIO, have also been reported [McCarthy et al., Multifunctional NANOAGENT FOR THROMBUS-TARGETED FIBRINOLYTIC THERApy. Nanomedicine. (Lond.) (Manuscript Submitted)]. Although these nanoagents have demonstrated increases in therapeutic efficacy, the analyses of the relevant safety profiles are yet to be undertaken and will be a major determinant of the utility of this class of materials.

\section{Bibliography}

1 Lanza GM, Wallace KD, Scott MJ et al. A novel site-targeted ultrasonic contrast agent with broad biomedical application. Circulation 94, 3334-3340 (1996).

2 Flacke S, Fischer S, Scott MJ et al. Novel MRI contrast agent for molecular imaging of fibrin: implications for detecting vulnerable plaques. Circulation 104, 1280-1285 (2001).

3 Yu X, Song SK, Chen J et al. High-resolution MRI characterization of human thrombus using a novel fibrin-targeted paramagnetic nanoparticle contrast agent. Magn. Reson. Med. 44, 867-872 (2000).

4 McCarthy JR, Patel P, Botnaru I, Haghayeghi P, Weissleder R, Jaffer FA. Multimodal nanoagents for the detection of intravascular thrombi. Bioconjug. Chem. 20, 1251-1255 (2009).

5 Jaffer FA, Tung CH, Wykrzykowska JJ et al. Molecular imaging of factor XIIIa activity in

\section{Conclusion}

Whereas nanomedicine has been mainly investigated for use in cancer diagnostics and therapeutics, as is evidenced by the number of publications in the field, it is well-positioned to play an increasingly larger role in cardiovascular disorders. As opposed to cancer, where there is great need to develop novel tools for its early diagnosis, thereby promoting improved outcomes, the diagnosis of thrombosis is routinely accomplished using a number of common clinical modalities. Given this, the nanoagents described above may not be utilized in front-line diagnostic imaging strategies, yet there is great value in their development to optimize patient-specific and thrombus biology-specific therapeutic regimes and hasten the next generation of targeted therapeutics with increased efficacy and safety.

\section{Financial \& competing interests disclosure}

This work was supported, in part, by an AHA Scientist Development Grant \# 0830352N (FAJ), Howard Hughes Medical Early Career Award (FAJ), NIH grants 1R01HL108229 (FAJ) and R21HL093607 (JRM) and NIH contract HHSN268201000044C (JRM). The authors have no other relevant affiliations or financial involvement with any organization or entity with a financial interest in or financial conflict with the subject matter or materials discussed in the manuscript apart from those disclosed.

No writing assistance was utilized in the production of this manuscript. thrombosis using a novel, near-infrared fluorescent contrast agent that covalently links to thrombi. Circulation 110, 170-176 (2004).

6 Ripplinger CM, Li C, Kim J, McCarthy JR, Lin CP, Jaffer FA. Multispectral in vivo molecular, functional, and anatomical imaging of venous thrombosis resolution. Arterioscleros. Throm. Vasc. Biol. 30, E183-E321 (2010).

7 Anderson HV, Willerson JT. Thrombolysis in acute myocardial infarction. $N$. Engl. J. Med. 329, 703-709 (1993).

8 Armstrong PW, Collen D. Fibrinolysis for acute myocardial infarction: current status and new horizons for pharmacological reperfusion, part 1. Circulation 103, 2862-2866 (2001).

9 No authors listed. Tissue plasminogen activator for acute ischemic stroke. The National Institute of Neurological Disorders and Stroke rt-PA Stroke Study
Group. N. Engl. J. Med. 333, 1581-1587 (1995).

10 Bi F, Zhang J, Su Y, Tang YC, Liu JN. Chemical conjugation of urokinase to magnetic nanoparticles for targeted thrombolysis. Biomaterials 30, 5125-5130 (2009).

11 Ma YH, Wu SY, Wu T, Chang YJ, Hua MY, Chen JP. Magnetically targeted thrombolysis with recombinant tissue plasminogen activator bound to polyacrylic acid-coated nanoparticles. Biomaterials 30, 3343-3351 (2009).

12 Marsh JN, Senpan A, Hu G et al. Fibrin-targeted perfluorocarbon nanoparticles for targeted thrombolysis. Nanomedicine (Lond.) 2, 533-543 (2007).

13 Marsh JN, Hu G, Scott MJ et al. A fibrin-specific thrombolytic nanomedicine approach to acute ischemic stroke. Nanomedicine (Lond.) 6, 605-615 (2011). 